The Astrophysical Journal, 645:1272-1282, 2006 July 10

(C) 2006. The American Astronomical Society. All rights reserved. Printed in U.S.A.

\title{
DISCOVERY OF AN OPTICALLY THICK, EDGE-ON DISK AROUND THE HERBIG Ae STAR PDS 144N ${ }^{1}$
}

\author{
Marshall D. Perrin \\ Astronomy Department, University of California, Berkeley, CA 94720-3411; mperrin@astro.berkeley.edu \\ GASPARD DUCHÊNE \\ Laboratoire d'Astrophysique, Observatoire de Grenoble, BP 53, F-38041 Grenoble, Cedex 9, France \\ AND \\ Paul Kalas and James R. Graham \\ Astronomy Department, University of California, Berkeley, CA 94720-3411 \\ Received 2005 October 11; accepted 2006 March 23
}

\begin{abstract}
We have discovered an optically thick, edge-on circumstellar disk around a Herbig Ae star in the binary system PDS 144, providing the first intermediate-mass analog of HK Tau and similar T Tauri stars. This system consists of a $V \sim 13$ mag primary and a fainter companion, with the spectra of both stars showing evidence for circumstellar disks and accretion; both stars were classified as Herbig Ae by the Pico dos Dias Survey. In Lick adaptive optics polarimetry, we resolved extended polarized light scattered from dust around the northern star. Follow-up Keck adaptive optics and mid-infrared observations show that this star is entirely hidden by an optically thick disk at all wavelengths from 1.2 to $11.7 \mu \mathrm{m}$. The disk major axis subtends $\sim 0$ ".8 on the sky, corresponding to $\sim 800$ AU at a distance of $1000 \mathrm{pc}$. Bright "wings" extend 0"3 above and below the disk ansae, due most likely to scattering from the edges of an outflow cavity in a circumstellar envelope. We discuss the morphology of the disk and the spectral energy distributions of the two PDS 144 stars, present preliminary disk models, and identify a number of open questions regarding this fascinating system.
\end{abstract}

Subject headings: accretion, accretion disks - circumstellar matter — dust, extinction planetary systems: protoplanetary disks - stars: individual (PDS 144) stars: pre-main-sequence

\section{INTRODUCTION}

Circumstellar disks inescapably form around young stars, as angular momentum conservation prevents infalling matter from accreting directly onto the stellar surface. Transfer of angular momentum between different parcels of matter in these disks not only allows continued accretion, but has been implicated in the launching of spectacular bipolar jets and winds (Shu et al. 1994). Studying the detailed physics of such disks is thus important for understanding the nature of these outflows, which provide feedback into the star formation process and help limit the star formation rate. The standard model for $\mathrm{T}$ Tauri stars invokes magnetic fields to drive the outflows. While strong magnetic fields are inevitable in convective, low-mass stars, the intermediate-mass Herbig Ae/Be stars (Herbig 1960; Waters \& Waelkens 1998) have much shallower convective zones and consequently have much weaker dynamos. Thus, it is natural to suppose that disk environments around these stars may differ substantially from those around T Tauri stars.

Characterizing these disks also illuminates the conditions in which planet formation takes place. The tremendous diversity of recently discovered extrasolar planetary systems raises the question of whether this diversity arises from widely differing initial conditions in circumstellar disks, or if instead all solar systems begin similarly but evolve apart via planetary dynamics. Are

\footnotetext{
1 Some of the data presented here were obtained at the W. M. Keck Observatory, which is operated as a scientific partnership among the California Institute of Technology, the University of California, and the National Aeronautics and Space Administration. The Observatory was made possible by the generous financial support of the W. M. Keck Foundation.
}

conditions around A- and B-type stars as conducive to planet formation as are those around lower mass stars? Present Doppler planet searches generally exclude A and B stars due to their relatively featureless spectra (although see Galland et al. [2005] for an exception to this rule), yet the presence of debris disks around the A stars Vega (Aumann et al. 1984) and $\beta$ Pic (Smith $\&$ Terrile 1984) provided the first evidence for the existence of extrasolar planetary systems. Indeed, more recent observations of perturbations to the disks of Vega and Fomalhaut indicate the presence of Jupiter-mass planets around those stars (Wilner et al. 2002; Kalas et al. 2005). The Herbig Ae/Be stars (Herbig 1960) are the progenitors of Vega-like debris disks. By studying the circumstellar material around these stars, we aim to quantify the evolution of dust properties and spatial distribution with stellar age and thereby gain insight into the growth of larger dust particles and, ultimately, planets.

Optically thick, edge-on disks are particularly amenable to spatially resolved observations in the visible or infrared. Disk imaging is frequently complicated by point-spread function (PSF) subtraction artifacts, especially on small angular scales. For optically thick, edge-on disks, the lack of direct starlight greatly reduces the dynamic range needed to image the disk. At the same time, modeling the observed light distribution can constrain the disk's physical parameters, such as the degree of flaring and dust grain growth (D'Alessio et al. 2001; Watson \& Stapelfeldt 2004). Edge-on disks are particularly valuable for such studies, as the disk's vertical structure is directly apparent. By modeling multiwavelength observations of edge-on disks, one can determine the wavelength dependence of the dust-scattering opacity, which provides insight into dust grain properties (Cotera et al. 2001; 
TABLE 1

OBSERVATION Log

\begin{tabular}{ccccrc}
\hline \hline Telescope & Instrument & Filter & Date & $\begin{array}{r}t_{\text {int }} \\
(\mathrm{s})\end{array}$ & $\begin{array}{c}\theta \\
(\operatorname{arcsec})\end{array}$ \\
\hline Lick 3 m ........ & \multirow{2}{*}{ IRCAL } & $J$ & 2004 Jul 5 & 320 & 0.38 \\
& & $H$ & 2004 Jul 5 & 480 & 0.33 \\
& & $K_{s}$ & 2004 Jul 5 & 240 & 0.30 \\
Keck II .......... & \multirow{2}{*}{ NIRC2 } & $H$ & 2004 Aug 29, 30 & 120 & 0.055 \\
& & $K^{\prime}$ & 2004 Aug 29 & 90 & 0.058 \\
& & PAH & 2004 Aug 30 & 144 & 0.07 \\
& & $L^{\prime}$ & 2004 Aug 30 & 90 & 0.08 \\
Keck I ........... & LWS & 11.7 $\mu \mathrm{m}$ & 2004 Aug 28 & 85 & 0.35 \\
\hline
\end{tabular}

Notes.-Observing $\log$ for PDS $144 ; t_{\text {int }}$ is the total integration time in each filter, and $\theta$ is the achieved resolution in arcseconds, measured from a point source. See Table 2 for a detailed description of filter properties.

Watson \& Stapelfeldt 2004). Edge-on disks in binary systems may additionally shed light on aspects of the binary formation process and disk-disk interactions.

Edge-on disks are known around a number of lower mass $\mathrm{T}$ Tauri stars, most notably HH 30 (Burrows et al. 1996), HK Tau B (Stapelfeldt et al. 1998), and HV Tau C (Monin \& Bouvier 2000), but until now no edge-on disk has been seen around a Herbig Ae or Be star. We present in this paper our discovery of such an edgeon circumstellar disk around the Herbig Ae star PDS 144N ( $\alpha=$ $\left.15^{\mathrm{h}} 49^{\mathrm{m}} 15^{\mathrm{s}} 5, \delta=-26^{\circ} 00^{\prime} 50^{\prime \prime} 2, \mathrm{~J} 2000.0\right)$.

Carballo et al. (1992) identified IRAS 15462-2551 with a previously anonymous 12th magnitude star in Scorpius. The Pico dos Dias Survey, a spectroscopic follow-up to Infrared Astronomical Satellite (IRAS) sources (Torres et al. 1995), subsequently named this source PDS 144 , discovered it to be a $5^{\prime \prime}$ binary, and classified both members as Herbig Ae stars. Vieira et al. (2003) reported a spectral type of A2 IV for the northern member and $\mathrm{A} 5 \mathrm{~V}$ for the southern member. We follow their notation in referring to these stars as PDS $144 \mathrm{~N}$ and PDS 144S, respectively. In addition to the $\mathrm{H} \alpha$ emission signature of ac- cretion, they found [S II] $\lambda \lambda 6717,6730$ and [O II] $\lambda \lambda 3726,3728$ emission around both stars, indicative of a photodissociation region. These lines are good tracers of outflows around other young stars, and their presence makes it probable that such outflows are present around both PDS 144 stars. The physical association of these two stars has not yet been rigorously shown, but given their proximity and the fact that both are Herbig Ae stars that are currently accreting and thus of similar age, it is very likely that they are indeed bound and coeval.

We describe our observations in $\S 2$ and discuss the distance to PDS 144 in $\S 3$. In $\S 4$ we examine the observed disk's morphology, before presenting preliminary models for the disk in $\S 5$. Finally, $\S 6$ recapitulates our conclusions and identifies a number of remaining open questions about this intriguing system.

\section{OBSERVATIONS}

PDS 144N's disk was discovered in observations obtained with Lick Observatory's $3 \mathrm{~m}$ Shane telescope and was subsequently imaged with both $10 \mathrm{~m} \mathrm{~W}$. M. Keck telescopes. Together, these observations span the wavelength range 1.2-11.7 $\mu \mathrm{m}$. An observation $\log$ is presented in Table 1, while Table 2 gives our measured photometry for both PDS 144 stars.

\subsection{Lick}

We first observed PDS 144 as part of our ongoing adaptive optics polarimetry survey of Herbig Ae/Be stars (Perrin et al. 2004a) being carried out with the Lick Observatory Adaptive Optics (AO) system (Gavel et al. 2002) and the IRCAL camera (Lloyd et al. 2000). IRCAL's differential polarimetry mode incorporates a Wollaston prism and rotating half-wave plate to obtain high-contrast images in polarized light (Perrin et al. 2004b). For these observations, the AO system was locked on PDS 144S. The seeing was excellent (Fried's parameter $r_{0} \sim 16 \mathrm{~cm}$ at $550 \mathrm{~nm}$ ), but the target was observed at an air mass of 2.25 , and $\mathrm{AO}$ correction was limited to a Strehl ratio of 0.1 at $K_{s}$ band. After dark subtraction and flat fielding, the polarimetric data were reduced via a double-differencing code based on the algorithm of

TABLE 2

Measured Fluxes

\begin{tabular}{|c|c|c|c|c|c|c|c|}
\hline Instrument & Filter & $\begin{array}{c}\lambda \\
(\mu \mathrm{m})\end{array}$ & $\begin{array}{c}\text { FWHM } \\
(\mu \mathrm{m})\end{array}$ & $\begin{array}{l}\text { PDS } 144 \mathrm{~N} \\
(\mathrm{Jy})\end{array}$ & $\begin{array}{l}\text { PDS 144S } \\
\text { (Jy) }\end{array}$ & $\begin{array}{l}\text { PDS } 144 \mathrm{~N} \\
(\mathrm{mag})\end{array}$ & $\begin{array}{l}\text { PDS 144S } \\
\text { (mag) }\end{array}$ \\
\hline \multirow[t]{3}{*}{ 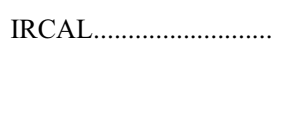 } & $J$ & 1.238 & 0.267 & $0.014 \pm 0.002$ & $0.098 \pm 0.012$ & $12.61 \pm 0.07$ & $10.53 \pm 0.06$ \\
\hline & $H$ & 1.650 & 0.297 & $0.025 \pm 0.003$ & $0.357 \pm 0.026$ & $11.52 \pm 0.06$ & $8.64 \pm 0.05$ \\
\hline & $K_{s}$ & 2.150 & 0.320 & $0.049 \pm 0.006$ & $1.343 \pm 0.102$ & $10.31 \pm 0.10$ & $6.71 \pm 0.10$ \\
\hline \multirow[t]{4}{*}{ 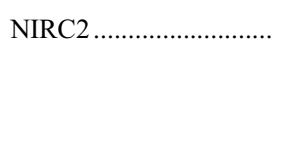 } & $H$ & 1.633 & 0.296 & $0.023 \pm 0.003$ & $>0.228 \pm 0.031$ & $11.64 \pm 0.02$ & $<9.15 \pm 0.02$ \\
\hline & $K^{\prime}$ & 2.124 & 0.351 & $0.043 \pm 0.004$ & $0.997 \pm 0.118$ & $10.48 \pm 0.02$ & $7.06 \pm 0.02$ \\
\hline & PAH & 3.290 & 0.056 & $0.277 \pm 0.034$ & $>1.576 \pm 0.196$ & $7.58 \pm 0.05$ & $<5.70 \pm 0.05$ \\
\hline & $L^{\prime}$ & 3.776 & 0.700 & $0.094 \pm 0.013$ & $>1.132 \pm 0.161$ & $8.56 \pm 0.04$ & $<5.85 \pm 0.04$ \\
\hline LWS . & 11.7 & 11.698 & 1.104 & $1.352 \pm 0.249$ & $3.751 \pm 0.692$ & $3.28 \pm 0.10$ & $2.17 \pm 0.10$ \\
\hline \multirow[t]{4}{*}{ IRAC } & 3.6 & 3.550 & 0.75 & $0.188 \pm 0.010$ & $1.97 \pm 0.099$ & $7.94 \pm 0.06$ & $5.39 \pm 0.06$ \\
\hline & 4.5 & 4.439 & 1.01 & $0.182 \pm 0.009$ & $2.44 \pm 0.123$ & $7.49 \pm 0.06$ & $4.67 \pm 0.06$ \\
\hline & 5.8 & 5.731 & 1.42 & $0.767 \pm 0.039$ & $2.33 \pm 0.118$ & $5.44 \pm 0.06$ & $4.23 \pm 0.06$ \\
\hline & 8.0 & 7.872 & 2.93 & $1.74 \pm 0.089$ & $2.49 \pm 0.127$ & $3.92 \pm 0.06$ & $3.53 \pm 0.06$ \\
\hline \multirow[t]{2}{*}{ 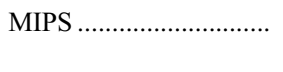 } & 24 & 23.7 & 4.7 & $3.74 \pm 0.190$ & $5.15 \pm 0.260$ & $0.70 \pm 0.06$ & $0.35 \pm 0.06$ \\
\hline & 70 & 71.4 & 19 & $2.58 \pm 0.140$ & $1.73 \pm 0.100$ & $-1.31 \pm 0.06$ & $-0.88 \pm 0.06$ \\
\hline
\end{tabular}

Notes.-Photometry for PDS 144. The IRCAL and NIRC2 measurements used a $1^{\prime \prime}$ radius aperture, while 1 ".7 was used for LWS. Aperture correction factors were computed using the Keck AO PSF model of Sheehy et al. (2006) and are typically $2 \%-4 \%$. Flux calibrations are as discussed in the text; the filter wavelengths listed above are isophotal wavelengths computed according to the method of Cohen et al. (1999). Columns marked with a $<$ or $>$ are limits derived from saturated observations of PDS 144S. The flux uncertainties in Jy include contributions from both the aperture photometry and the absolute flux calibration. For Spitzer data, filter properties are taken from Fazio et al. (2004) and Rieke et al. (2004). Photometry and statistical uncertainties were computed with the APEX package, plus an estimated $5 \%$ absolute flux calibration error for all Spitzer bands. 


\section{Total Intensity}
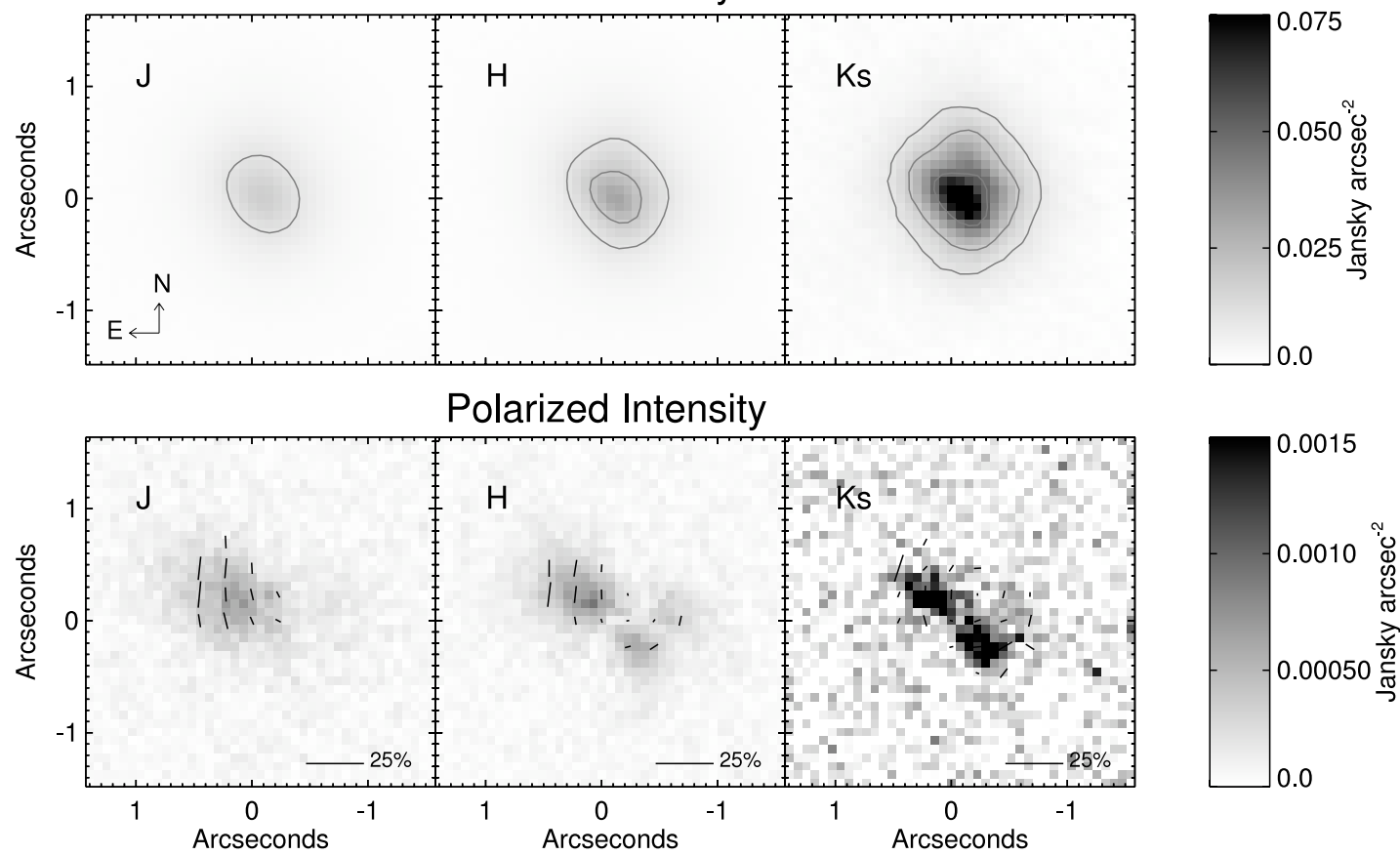

FIG. 1.-Lick AO polarimetry observations of PDS $144 \mathrm{~N}$. The top row shows total intensity $I$, while the second row shows polarized intensity $\left(Q^{2}+U^{2}\right)^{1 / 2}$, with position

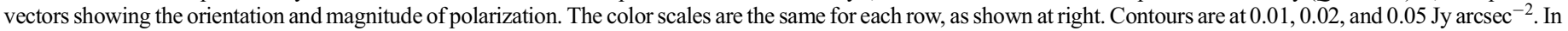

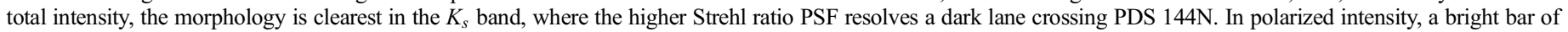

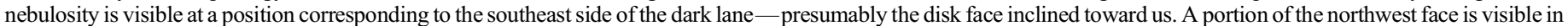

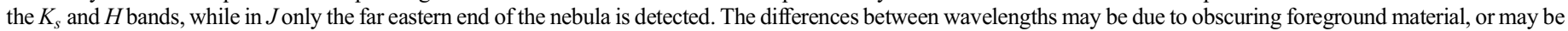

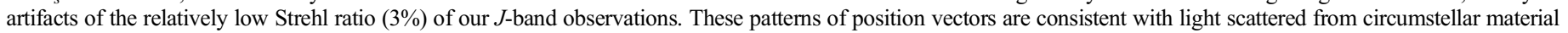
around PDS 144N.

Kuhn et al. (2001), resulting in images of the Stokes parameters $I, Q$, and $U$ for each near-infrared band. These data were then flux calibrated using the standards HD 161903 (Elias et al. 1982) and [PMK98] 9170 (Persson et al. 1998).

The northern source is resolved at all wavelengths, while the southern source is pointlike (Fig. 1). At $H$ and especially $K_{S}$ band, PDS $144 \mathrm{~N}$ appears as an elliptical nebula bisected by a dark lane. In polarized light, PDS $144 \mathrm{~N}$ is extended $\sim 1^{\prime \prime}$ from northeast to southwest, with polarization position vectors roughly perpendicular to this. Centrosymmetric polarization vectors are the signature of scattered starlight, and the presence of such vectors along one axis is precisely what is expected for an edgeon circumstellar disk. The observed polarization fractions range between $5 \%$ and $10 \%$, although this is a lower limit for the true polarization, since the observed values are biased downward by AO-uncorrected light.

In polarized light, PDS $144 \mathrm{~S}$ appears only at the $1 \%-2 \%$ level of systematic subtraction residuals expected for an unpolarized source. The position vectors are not centrosymmetric, and based on these polarization data alone there is no direct evidence for a disk around PDS 144S.

\subsection{Keck}

Shortly after the disk was discovered at Lick, we obtained additional near-infrared data with the Keck II AO system (Wizinowich et al. 2000) and NIRC2 camera (K. Matthews 2006 , in preparation). PDS 144 S again served as the wave-front reference. The Keck images have much better AO correction than the Lick data, in part because PDS 144 is observable at a more favorable air mass from Keck (Figs. 2 and 3). Because the exposure times were chosen to maximize signal-to-noise ratio $(\mathrm{S} / \mathrm{N})$ on the PDS $144 \mathrm{~N}$ disk, PDS $144 \mathrm{~S}$ is saturated in all wave- lengths other than $K^{\prime}$. These imaging data were reduced via the usual steps of dark and sky subtraction, flat fielding, and mosaicking, and were flux calibrated using the same photometric standard, [PMK98] 9170. For the $3.3 \mu \mathrm{m}$ polycyclic aromatic hydrocarbon (PAH) filter, no photometric standard observations were available, nor was a typical zero point available from the NIRC2 documentation. We therefore estimated a zero point for the PAH filter relative to our measured zero points for the other filters, using the known PAH filter response, detector quantum efficiency, and the typical Mauna Kea atmospheric transmission across the filter bandpass (Roe 2002). To evaluate the reliability of this synthetic PAH zero point, we similarly estimated a $K^{\prime}$ zero point; the estimated and measured $K^{\prime}$ zero points agreed within 0.05 mag.

The center of PDS $144 \mathrm{~N}$ is located 5".40 \pm 0 . 01 from PDS $144 \mathrm{~S}$, at a position angle of $28^{\circ} .7 \pm 0.3$. It is difficult to define an exact center for an extended, obscured object; neither the location of peak brightness nor the photocenter is appropriate, so we used a position roughly in the middle of the dark lane but displaced slightly toward the brighter face. Based on our disk models discussed below, this is a reasonable estimate for the location of the hidden star.

In addition to the two known members of PDS 144, we detect three other point sources within our full field-of-view mosaics (roughly $13^{\prime \prime} \times 11^{\prime \prime}$ ). Photometry and astrometry for these sources are given in Table 3. Most notably, there is a faint source located almost directly between the two stars of PDS 144. These are most likely field stars, either foreground or background. If so, and assuming they are not high proper motion foreground objects, they may be useful as astrometric references for PDS 144. Its

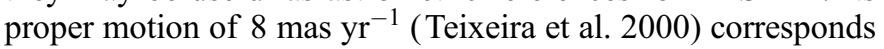
to one $K_{s}$-band Keck resolution element per 4 years, so propermotion tests will be possible rapidly. 

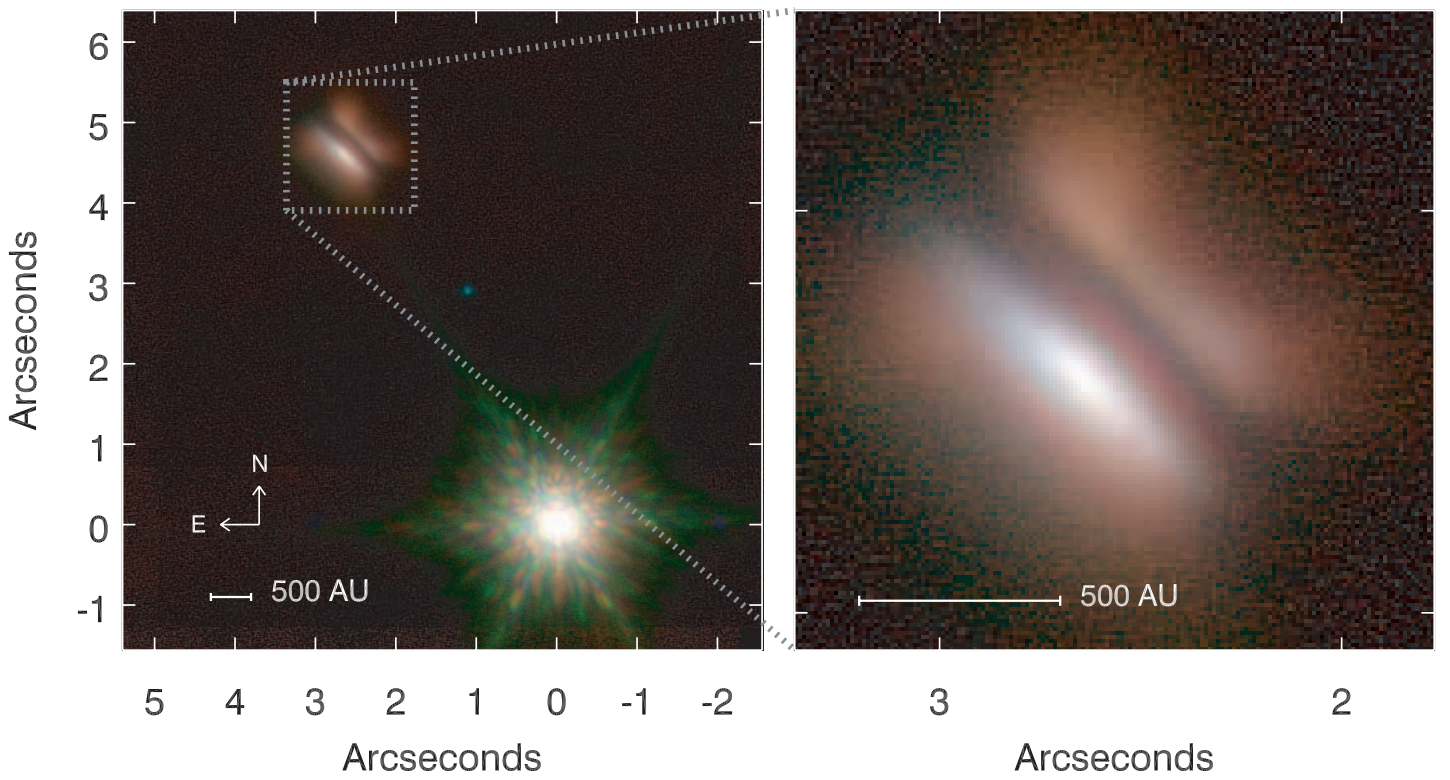

FIG. 2.-Keck AO imaging of PDS 144. The $H, K^{\prime}$, and $L^{\prime}$ bands are shown as blue, green, and red, respectively. PDS $144 \mathrm{~N}$ appears as an edge-on disk seen in silhouette against brighter nebulosity, which extends vertically on both sides of the disk plane. Both PDS 144N and the brighter PDS 144S are Herbig Ae stars, while the nature of the faint, third source located almost directly between them is unknown. The scale bar in AU assumes a distance of $1000 \mathrm{pc}$; see $\S 3$. The nebulosity around PDS $144 \mathrm{~N}$ flares vertically near its edges, reminiscent of a pair of opposing wingnuts. In the northwestern, fainter face of the nebula, the wings are sufficiently prominent that the nebula is brighter toward its outer edges than in the middle: there is a local minimum in intensity on the symmetry axis. The presence of a circumstellar envelope in addition to a disk is required to explain these "wings."

During the same observing run, we also obtained a mid-infrared image with the Long Wavelength Spectrometer (LWS; Jones \& Puetter 1993) on Keck I (Fig. 3). LWS was used with the $11.7 \mu$ m filter in chop-nod imaging mode, with a $10^{\prime \prime}$ chop throw at a position angle of $0^{\circ}$ and a chop frequency of $2.5 \mathrm{~Hz}$. Data were reduced using the standard double-difference subtraction to remove sky, telescope, and instrumental backgrounds; no flat fielding was performed. The photometric standards HD 197989 and HD 2486 (Cohen et al. 1999) were used to flux calibrate the data. We rotated and rebinned the LWS data to register them with our NIRC2 data, using the documented plate scales of 9.94 and 84.7 mas pixel $^{-1}$, respectively. Image quality was near diffraction limited (FWHM 0.35 ). The LWS image shows morphology very similar to the near-IR data, particularly if the near-IR data are reduced to the same resolution as the LWS image.

\subsection{Spitzer}

Spitzer observations of PDS 144 are available from the Spitzer Archive. These observations, originally carried out as part of the
Molecular Cores to Planets legacy program (Evans et al. 2003), span 3.6-70 $\mu \mathrm{m}$ using the Infrared Array Camera (IRAC) and the Multiband Imaging Photometer (MIPS). We retrieved the pipelineprocessed Post-BCD mosaics from the archive and performed photometry with the APEX package (Makovoz \& Marleau 2005). APEX was able to successfully deblend the sources, even with the substantial PSF overlap at longer wavelengths. Since PDS 144 was the only source in the field, no contemporaneous PSF measurement was available, so we used the reference PSFs from the Spitzer Web site. The Spitzer photometry is presented in Table 2 and Figure 4.

We note in passing that the summed fluxes for PDS 144 in the MIPS $24 \mu \mathrm{m}$ band are in excellent agreement with the $20 \mu \mathrm{m}$ measurement by IRAS, which did not resolve the two sources. However, the $60 \mu \mathrm{m} I R A S$ flux is 3 times the MIPS $70 \mu \mathrm{m}$ measurement, indicating that the IRAS measurement at that wavelength suffers from confusion due to the large beam size. No other bright sources are present in the MIPS field of view, so it is unknown whether other source(s) or extended emission from diffuse gas are responsible for this confusion.

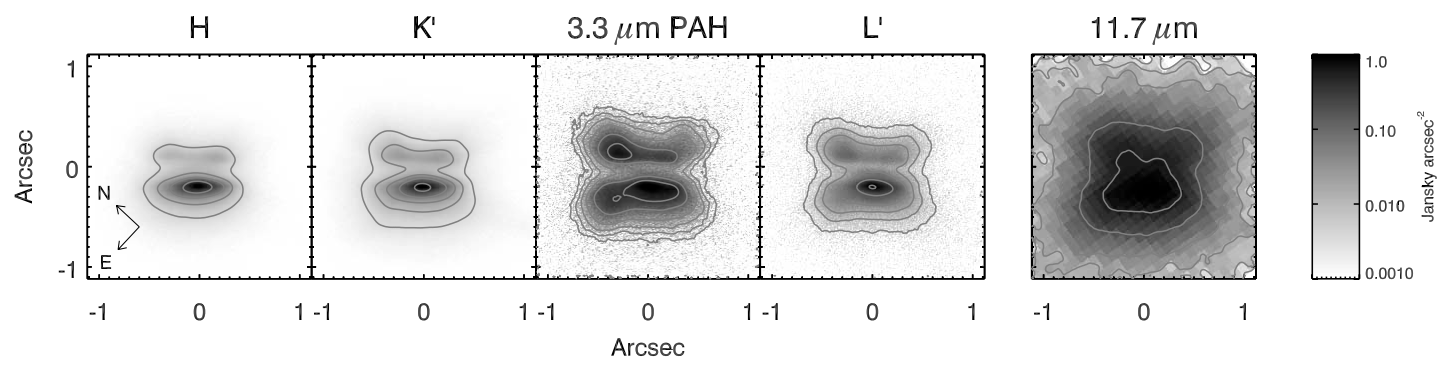

FIG. 3.-PDS 144N disk, as observed with NIRC2 on Keck II (left four frames) and LWS on Keck I (right frame). All are displayed with the same logarithmic intensity

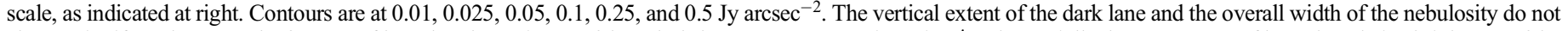

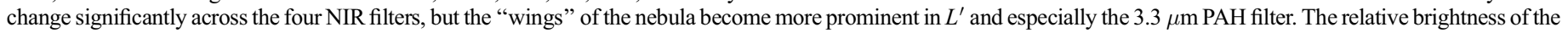

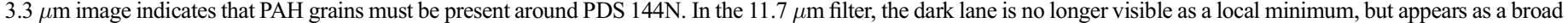

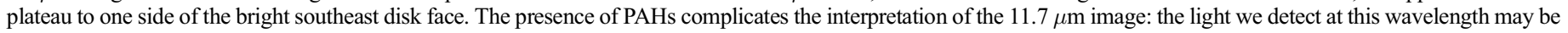

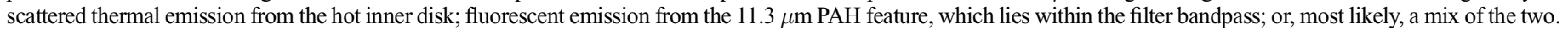


TABLE 3

Additional Sources Detected

\begin{tabular}{cccc}
\hline Source & $\begin{array}{c}\text { R.A. Offset } \\
(\operatorname{arcsec})\end{array}$ & $\begin{array}{c}\text { Decl. Offset } \\
(\operatorname{arcsec})\end{array}$ & $H$ Magnitude \\
\hline $3 \ldots \ldots \ldots \ldots \ldots \ldots \ldots . .$. & 1.122 & 3.000 & $15.50 \pm 0.10$ \\
& 7.768 & 3.480 & $16.55 \pm 0.20$ \\
\hline
\end{tabular}

Note.-R.A. and Decl. offsets are relative to PDS 144 S and have uncertainties of \pm 0.005 .

\subsection{Spectral Energy Distribution}

The spectral energy distribution (SED) for the PDS 144 system is shown in Figure 4. In addition to our own measurements, we plot the optical points from Vieira et al. (2003). We overplot flux-calibrated stellar spectra corresponding to the spectral types of PDS 144: A5 and A2 for PDS 144N and PDS 144S, respectively. We assume here a distance of $1 \mathrm{kpc}$, but see $\S 3$ for a detailed discussion of the distance to PDS 144, which remains very uncertain.

PDS 144S's SED rises sharply from 1 to $2 \mu \mathrm{m}$ before leveling off, while PDS 144N's SED is nearly flat throughout the optical and near-IR. They are both therefore class II Herbig Ae stars in the classification system of Hillenbrand et al. (1992). Other Herbig Ae stars with SEDs similar to PDS $144 \mathrm{~N}$ include $\mathrm{LkH} \alpha$ 233 and Parsamian 21. Those sources are both embedded deeply enough that the stars are completely hidden at optical and nearIR wavelengths and only scattered light is seen (Staude \& Neckel 1992; Perrin et al. 2004a), just as in PDS 144N.

At $70 \mu \mathrm{m}$, PDS $144 \mathrm{~N}$ is brighter than its southern neighbor. This suggests that while both stars possess substantial circumstellar dust, PDS 144N has a greater amount of cool dust at larger orbital radii than does PDS 144S.

\section{THE DISTANCE TO PDS 144}

The distance to PDS 144 remains frustratingly uncertain. Previous estimates in the literature range from 140 to $2000 \mathrm{pc}$. The nearer distance was suggested by Teixeira et al. (2000), who assumed that PDS 144 lies in the Upper Scorpius-Ophiuchus star-forming region, based primarily on its position in the sky. The more distant estimate is from Vieira et al. (2003), who derived an optical photometric distance of $2000 \mathrm{pc}$ for PDS 144N, and $1030 \mathrm{pc}$ for PDS 144S.

The lack of resolved optical photometry for PDS 144 complicates the determination of a photometric distance. Resolved optical photometry will be available from Hubble Space Telescope (HST) observations scheduled for the near future. Until then, we must make do with a best estimate from the currently available data. Our near-IR measurements are unsuitable for photometric distance measurements, since they are presumably contaminated by IR excess from circumstellar dust. We note that the $K^{\prime}$-band flux of PDS 144S is consistent with the photospheric flux from an A5 star located at $140 \mathrm{pc}$. Since the true $K^{\prime}$ luminosity is presumably greater than photospheric, the actual distance must be $\geq 140$ pc.

While Vieira et al. (2003) only reported combined photometry for PDS $144(V=12.8 \mathrm{mag})$, they measured $\Delta V=1.1 \mathrm{mag}$ from the relative counts of the two sources in their spectroscopic observations (C. Torres 2005, private communication). This implies $V=13.1$ and 14.2 for PDS $144 \mathrm{~S}$ and $144 \mathrm{~N}$, respectively. From these numbers, Vieira et al. derived their 1030 and $2000 \mathrm{pc}$ photometric distances. Since we see PDS 144N only in scattered

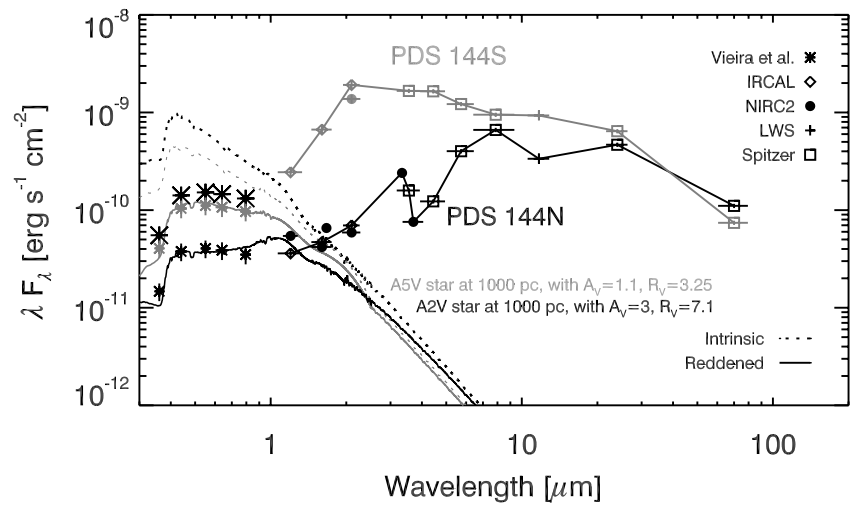

FIG. 4.-SED for PDS 144. The key at right indicates which instrument each plotted point is from. Horizontal bars indicate the FWHMs of each filter bandpass, while vertical bars (typically smaller than the plot symbols) show the uncertainties. For the optical points from Vieira et al. (2003), the larger asterisks (top) are their reported total fluxes for both stars of PDS 144, while the two rows of smaller symbols are our estimates for the two stars' individual fluxes, as discussed in $\S 3$. We overplot both intrinsic and reddened stellar spectra for the two stars. The A5 spectrum for PDS $144 \mathrm{~S}$ has been attenuated by $A_{V}=1.1$, $R_{V}=3.1$ using the extinction curve of Cardelli et al. (1989). Since PDS 144N is seen entirely in scattered light at optical wavelengths, it is a crude approximation to fit it with a reddened stellar spectrum. We show here an A2 spectrum reddened by $A_{V}=3, R_{V}=7.1$ to demonstrate that the observed optical magnitudes can be obtained with a moderate amount of dust, and caution that $A_{V}=3$ does not represent the direct line-of-sight extinction to the star through the highly optically thick disk midplane.

light, it seems certain that its $2000 \mathrm{pc}$ photometric distance is an overestimate. But the photometric distance for the less obscured PDS 144S may be accurate. Given PDS 144S's unresolved appearance, it is plausible that we see its photosphere directly; certainly it is not as obscured as PDS 144N. If we assume this is the case, then we can follow Vieira et al. (2003) and derive a photometric distance to PDS 144S.

We estimated the optical fluxes of PDS 144S from the combined $U B V R I$ magnitudes and $\Delta V$ from Vieira et al., assuming that the flux ratio between the two sources is the same for all wavelengths. (Although this assumption is crude, the following results are only weakly dependent on it.) We then performed a nonlinear least-squares fit to PDS 144S using a flux-calibrated zero-age main sequence (ZAMS) A5 V stellar spectrum, reddened by dust using the extinction law of Cardelli et al. (1989) and then multiplied by appropriate filter bandpasses. The free parameters of the fit were the distance $d$, the visual extinction $A_{V}$, and the reddening parameter $R_{V}$; we assume there is only one dust component along the line of sight. The best-fit parameters are $d=1030 \pm 130 \mathrm{pc}, A_{V}=1.1 \pm 0.46$, and $R_{V}=3.25 \pm 0.65$ (see Fig. 5). These formal errors underestimate the true uncertainty, given the various assumptions that went into the model. Changes in the assumed flux ratio between PDS 144S and 144N can shift the resulting best-fit distance by an additional $\sim 100 \mathrm{pc}$. Future work is certainly needed to pin down the distance to PDS 144 more precisely. Nonetheless, for the moment we adopt $1000 \pm 200 \mathrm{pc}$ as a working estimate.

A major assumption in this distance fit is that PDS 144S has luminosity for an A5 star on the ZAMS. Many Herbig Ae/Be stars have luminosities a few times greater than ZAMS values. If PDS 144 is significantly pre-main sequence and thus more luminous than we assume, it will be correspondingly more distant, which will only exacerbate the problem of its height above the Galactic plane (see below).

Another way to estimate the distance to PDS 144 is by comparison with other Herbig Ae stars of known distance and similar 


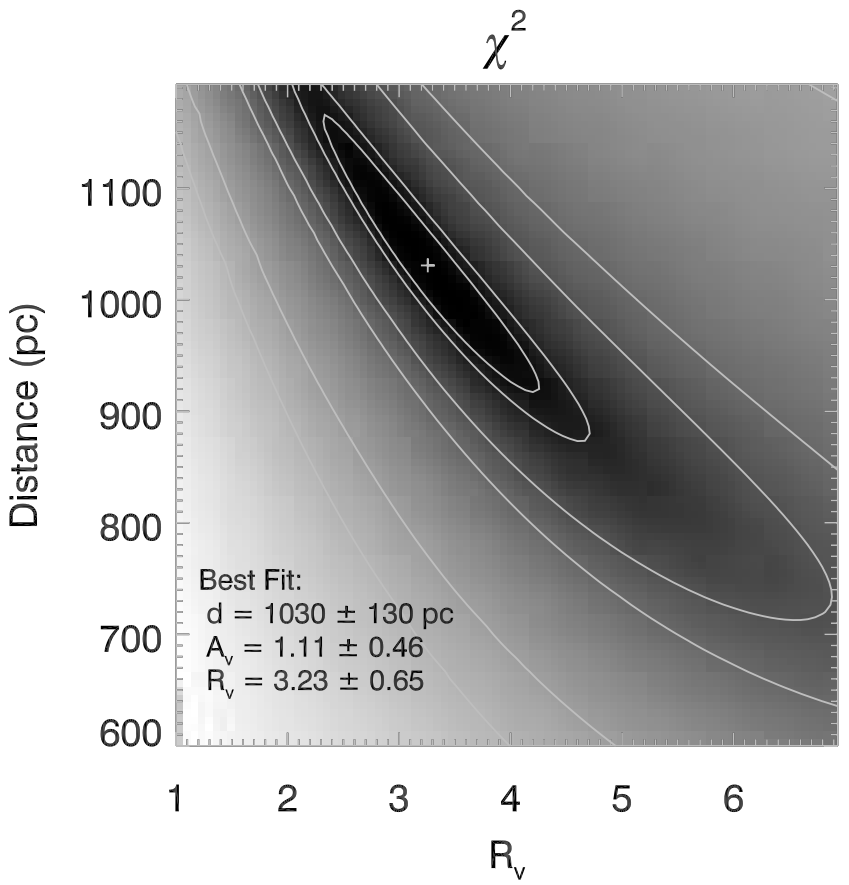

FIG. 5.-Synthetic UBVRI photometry fit to PDS $144 \mathrm{~S}$ in order to estimate its distance, as described in the text; plotted here is the resulting $\chi^{2}$ against distance and reddening. Vieira et al. (2003) did not provide uncertainty for their photometry; not knowing the error bars results in our $\chi^{2}$ being uncertain by an arbitrary multiplicative constant. We therefore normalize our best-fit $\chi^{2}=1$ and plot here contours of $0.5,1,5,10,50$, and 100 above the best-fit $\chi^{2}$. Our best fit, marked by a plus sign, gives a distance of $1030 \mathrm{pc}$. While there is substantial covariance between the distance and the extinction law parameters, distances less than $\sim 500$ pc do not appear plausible.

spectral type. This has the advantage of not requiring us to assume a particular luminosity for PDS 144S. For instance, IP Per is an A6 star with $V=10.5$ at a distance of 350 pc (Hernández et al. 2004). For it to have the same apparent brightness as PDS 144 S, IP Per would need to be located at $1200 \mathrm{pc}$, assuming the extinction is unchanged. Different results are possible, depending on which star is picked as the analog of PDS 144S. Using a sample of 10 A4-A6 Herbig Ae stars taken from the literature, this procedure results in distances of $550-1900$ pc. It is encouraging that our $1000 \mathrm{pc}$ estimate falls in the middle of this range, but these comparisons do not reduce our uncertainty in the distance to PDS 144.

These data seem to rule out any association of PDS 144 with Upper Scorpius at $140 \mathrm{pc}$. Even with extraordinarily gray dust $\left(R_{V}>20\right)$, models of PDS $144 \mathrm{~S}$ with a distance of $140 \mathrm{pc}$ and sufficient extinction to produce $V=13.1$ predict $V-R \approx 1$, inconsistent with the observed $V-R=0.30$. Known Herbig Ae stars at the distance of Upper Scorpius are either some 3-5 mag brighter at $V$ than PDS 144 (e.g., KK Oph, HD 163296, or TY $\mathrm{CrA}$ ) or are embedded objects much redder than PDS 144 (e.g., Elias 1, which has $V-R=2.1$; Herbst \& Shevchenko 1999). A larger distance also provides a natural explanation for the low

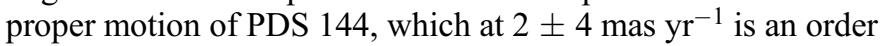
of magnitude slower than the average for Upper Scorpius/ Ophiuchus (Teixeira et al. 2000; Ducourant et al. 2005).

But a $1 \mathrm{kpc}$ distance is not without problems of its own. Chief among these is how did such a young system come to be so far above the Galactic plane? At a Galactic latitude of $+21^{\circ}$ and a distance of $1000 \pm 200 \mathrm{pc}$, PDS 144 is $360 \pm 70$ pc above the Galactic plane. Kroupa (1998) claimed that stars of all masses can be ejected at high velocities from moderate to dense clusters, so perhaps PDS 144 is a runaway from a star-forming region closer to the plane. But if that is the case, it is difficult to understand how the binary would have remained bound, since the necessary velocity to reach its current position in less than a few million years $\left(50-100 \mathrm{~km} \mathrm{~s}^{-1}\right)$ is greatly in excess of the binary orbital velocity, $\left.\approx 1 \mathrm{~km} \mathrm{~s}^{-1}\right)$. Further contradicting this scenario

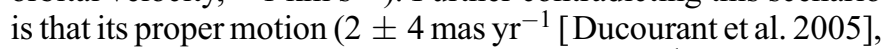
corresponding to a transverse velocity of $9 \mathrm{~km} \mathrm{~s}^{-1}$ at $1000 \mathrm{pc}$ ), is both slow and directed mostly toward the Galactic plane. We are left with the conclusion that this system must have formed far above the Galactic plane. It is unknown what star-forming region may have given birth to this system; no dark clouds are found within $2^{\circ}$ of PDS 144 according to the catalog of Dobashi et al. (2005).

One possible resolution to this dilemma would be if we have entirely misinterpreted the nature of these stars, and that they are in fact evolved post-main-sequence objects rather than young stars. Protoplanetary nebulae appear similar to pre-main-sequence A stars in several aspects, such as IR excess from dust, the presence of PAH emission, and optical forbidden lines such as [S II]. The PDS catalog is known to be contaminated by such interlopers (for instance, PDS 461/Hen 3-1475; García-Hernández et al. 2002). There are two reasons we reject this interpretation. First, the morphology of PDS $144 \mathrm{~N}$ more closely resembles edge-on disk systems than it does protoplanetary nebulae such as PDS 461. Second, both stars in PDS 144 display IR excess and optical forbidden lines. Since their spectral types and presumably their masses differ, we would not expect them to both evolve off the main sequence simultaneously. It is much more plausible for the pair to be young stars approaching the main sequence together rather than departing it.

PDS 144 is by no means the only Herbig Ae/Be star whose distance has proved problematic. $\mathrm{LkH} \alpha 101$, long believed to be located at $800 \mathrm{pc}$, may instead lie only $140 \mathrm{pc}$ distant (Stine \& O’Neal 1998). Conversely, the distance to HD 34282 has recently been revised upwards from 160 to 350 pc (Piétu et al. 2003; Merín et al. 2004). Similar methods to those used in the just-cited papers may help clarify the distance to PDS 144. In particular, highresolution optical spectroscopy could constrain stellar properties directly, while millimeter observations of Keplerian rotation in the disk of PDS 144N could provide a dynamical distance.

\section{DISK MORPHOLOGY}

\subsection{Basic Appearance}

At all wavelengths up to $11.7 \mu \mathrm{m}$, the exciting star within PDS $144 \mathrm{~N}$ is hidden from direct view by the dark lane of an edge-on circumstellar disk. The dark lane is 0 "' 15 in height and extends $\sim 0$."8 in the orthogonal direction (Fig. 2). Bright scattered-light nebulosity is visible on both sides of the disk plane, with the southeast side being brighter and therefore nearer, assuming the grains are forward scattering.

At our assumed distance, the 0."8 apparent disk diameter corresponds to a physical diameter of $800 \pm 180$ AU. This is comparable to disks around other Herbig Ae stars such as HD 100546 (500 AU) or HD 163296 (900 AU) (Grady et al. 2000, 2001, 2005). However, it is possible that the observed dark lane is actually a shadow cast by a smaller disk into a surrounding envelope (Pontoppidan \& Dullemond 2005). In this case the actual disk size could be smaller than the 800 AU apparent size. Pontoppidan \& Dullemond listed a number of observational criteria that can be used to discriminate disk shadows from large disks, such as the presence of a bright point source in the middle of the dark lane or a "wedgelike" shape, but none of these criteria fit PDS $144 \mathrm{~N}$. 
PDS $144 \mathrm{~N}$ is laterally asymmetric. In the inner region of the nebula, the peak intensity is displaced 0.05 southwest from the vertical axis. In the outer nebula, the northeast side is more extended than the southwest. Similar asymmetries in the disk of $\mathrm{HH} 30$ proved to be variable over timescales of a few years and have been interpreted as changing illumination caused by the orbital motion of clumps at a few AU (Stapelfeldt et al. 1999). Alternatively, those asymmetries may be evidence for a hot spot on the rotating stellar surface (Cotera et al. 2001). Long-term monitoring of PDS $144 \mathrm{~N}$ is needed to see whether its lateral asymmetries also vary with time. If so, then the timescales and amplitude of the variability may clarify whether it is driven by an outflow (such as by a precessing jet), a hot spot, shadowing by orbiting dust clumps, or some other mechanism. Alternatively, the asymmetry in PDS $144 \mathrm{~N}$ could be due to disk inhomogeneities at large radii where orbital periods are many decades, and so we may never see any changes.

\subsection{The "Wings"}

One of the most striking features of the nebulosity around PDS $144 \mathrm{~N}$ is the pronounced flaring at its outside edges: it resembles an opposing pair of wingnuts, with the outer vertical extensions being the "wings." The wings begin approximately 0 " 15 on either side of the disk's vertical axis and extend 0"4 above and below the disk midplane, twice the extent of the scattered light near the axis. The wings are comparatively redder than the inner portions of the nebula; they are most pronounced in the PAH and $L^{\prime}$ filters, but are still visible at a lower level in $H$ and $K^{\prime}$ (Fig. 3).

Hollenbach et al. (1994) proposed that massive stars can launch disk winds by photoevaporating the outer portions of circumstellar disks. Could such winds boiling off the disk be responsible for creating the wingnut morphology? The predicted wind emerges from the outer disk along outward-curving streamlines, which resemble the wings around PDS 144. The characteristic radius at which such evaporation is expected to take place is $r_{\text {evap }}=G M_{*} / a_{s}^{2} \simeq 7 \mathrm{AU}\left(M_{*} / M_{\odot}\right)$, where $a_{s}$ is the sound speed in the disk. But at a $1 \mathrm{kpc}$ distance, $r_{\text {evap }} \approx 0.02$, an order of magnitude smaller than the observed size, and we must find some other explanation for the wings.

Perhaps the young stellar object (YSO) that most resembles PDS 144N is IRAS 04302+2247 (Padgett et al. 1999), sometimes called the Butterfly Star in Taurus. Like PDS 144N, it has a dark lane bisecting two bright regions of nebulosity, each of which has its center fainter than its outer edges, giving an overall quadripolar morphology. Foreground extinction has been suggested as the cause of IRAS 04302+2247's quadripolar appearance, but we can rule this explanation out for PDS 144: the quadripolar appearance is still prominent at $11.7 \mu \mathrm{m}$ despite the much greater dust-penetrating ability of photons at that wavelength. Wolf et al. (2003) modeled IRAS 04302+2247 as a disk plus an infalling circumstellar envelope with bipolar outflow cavities. The cavity walls scatter more light than the evacuated cavities themselves, providing a natural explanation for the wings of the "butterfly"-and perhaps also the wings of PDS 144N. We present disk plus envelope models of PDS 144N in $\S 5$.

The wings become redder as one moves farther from the disk midplane. Intriguingly, this is opposite the blue color seen for the envelope around HD 100546 by Ardila et al. (2005). Possible explanations for the red color include, in decreasing order of likeliness:

1. The illuminating light scattered there is redder, due to greater contribution from the hot inner disk: grains in the en- velope see a larger solid angle subtended by the inner disk than do grains nearer the disk midplane.

2. Small grains in the envelope emit thermal radiation after being transiently heated to $\sim 600 \mathrm{~K}$.

3. Dust grains in the envelope might instead be larger than disk grains and thus scatter red light more efficiently; this seems unlikely, as it is inconsistent with our understanding of grain growth.

4. Scattered light from the envelope may experience more extinction than that from the disk. Some combination of factors 1 and 2 is most likely; these could be distinguished by future $L^{\prime}$ polarimetry observations.

\subsection{Disk Truncation?}

As in HV Tau C, PDS 144N's apparent diameter does not change significantly with wavelength. Since optical depth is a strong function of wavelength, this suggests that the circumstellar material around PDS 144N has a sharp outer edge, possibly because of tidal truncation by PDS 144S. The expected limit on disk size from tidal truncation is roughly $1 / 3$ of the binary's separation (Artymowicz \& Lubow 1994). If we assume that PDS 144's orbit is circular with the observed separation, $5400 \mathrm{AU}$, corresponding to the major axis, then the expected tidal truncation radius is $\approx 1800 \mathrm{AU}$, several times the observed radius. This is a lower limit on the truncation radius, since the true separation must be greater than or equal to the observed separation in the plane of the sky. Thus, while PDS 144 is not inconsistent with tidal interaction models, tides do not seem to be the limiting factor on disk size here.

However, a third source, such as a brown dwarf companion, might provide an alternative source of tides. We reiterate that there is a third, much fainter source located almost directly between the two PDS 144 stars (Table 3). Future observations are required to tell if this is a comoving member of the system or not. But given the hierarchical nature of most binaries and its position equidistant from both A stars, it is most likely merely a background star.

There is another possibility: the radiative effects of PDS 144S may be more important in truncating the disk than its gravity. FarUV photons from PDS 144S could heat and evaporate the outer disk, as has been suggested to occur for disks around low-mass stars externally illuminated by nearby massive stars (Adams et al. 2004). However, unlike typical proplyds in Orion, in which the external illuminating source is much brighter than the stars within the disks, in PDS 144 both sources are of comparable intrinsic luminosity. More detailed modeling is needed to disentangle the photoevaporative effects of the two stars.

We caution that the truncation of PDS 144N's disk and envelope by either tidal or photoevaporative forces is speculative. The presence of substantial IR excesses around both stars and the lack of any visible tidal streamers or other debris from a disrupted disk suggest that there is an alternative argument to be made for little interaction between the two stars.

\subsection{PAH Emission}

PAHs produce several IR emission features frequently seen around Herbig Ae stars (Leger et al. 1989; Natta \& Kruegel 1995). These emission bands arise due to fluorescent excitation of PAHs by far-UV photons, which are produced in great quantity by hot A stars.

PDS $144 \mathrm{~N}$ has emission several times brighter than continuum levels in the $3.3 \mu \mathrm{m}$ PAH band (Fig. 3). We used for the $3.3 \mu \mathrm{m}$ continuum a linear interpolation between the $2.1 \mu \mathrm{m} K^{\prime}$ and $3.8 \mu \mathrm{m}$ $L^{\prime}$ filters, neither of which contains the PAH feature within its 
bandpass. The PAH emission is detected over roughly the same region as is the continuum, but their relative intensities vary. As measured in NIRC2's $0.05 \mu \mathrm{m}$ wide PAH filter, the PAH emission is $\sim 6$ times brighter than continuum in the outer nebula, but in the bright core nearest the central star, the PAHs are only 2 times the continuum. In other words, the PAH emission is less centrally peaked than the continuum.

In order for PAH emission to occur, the PAHs must be illuminated by far-UV photons. Flared disks allow starlight to directly fall on PAHs in the disk surface, favoring PAH emission, while flat disks tend to lack PAH emission because PAHs are in the shadow of the inner disk (Acke \& van den Ancker 2004; Habart et al. 2006). PDS $144 \mathrm{~N}$ is consistent with this scenario: the PAH emission we see comes primarily from particles in the envelope, high above the disk's midplane. The models of Habart et al. (2004) predict that the $3.3 \mu \mathrm{m}$ PAH emission will be strongest within 100 AU of the star, a conclusion supported by recent observations. Here we detect that feature at several times greater distance. We find that the $\mathrm{PAH} /$ continuum ratio increases with distance from the star, as predicted by Habart et al. However, the models of Habart et al. were computed for face-on inclinations, so we cannot directly compare them with PDS 144N's edge-on geometry.

The Spitzer IRAC $8 \mu \mathrm{m}$ flux for PDS $144 \mathrm{~N}$ is significantly greater than the $11.7 \mu \mathrm{m}$ LWS flux. This suggests that PDS $144 \mathrm{~N}$ has strong PAH emission in the 7.7 or $8.6 \mu \mathrm{m}$ PAH bands as well, but higher spectral resolution observations are needed to confirm this.

\subsection{The Mid-Infrared}

What is the origin of the photons we detect from PDS 144N at $11.7 \mu \mathrm{m}$ ? The outer disk is too cool to emit thermal photons over the observed spatial extent. Nor can the light be scattered stellar photons, since the observed flux is far in excess of the photospheric value at this wavelength. However, it may be scattered photons from the hot inner disk, as is seen around HK Tau B at this wavelength (McCabe et al. 2003). PDS 144N must have substantial thermal emission from its inner disk (unless it has a large inner radius, which we cannot exclude based on our data) providing sufficient photons to scatter and produce the observed mid-IR nebulosity. Alternatively, it could be emission from the 11.3 $\mu \mathrm{m}$ PAH band, which falls within the bandpass of LWS's $11.7 \mu \mathrm{m}$ filter. The strength of the $11.3 \mu \mathrm{m}$ PAH feature should correlate with the $3.3 \mu \mathrm{m}$ feature, since both bands arise from the same aromatic $\mathrm{C}-\mathrm{H}$ bonds (Bregman et al. 1994), and we know there is copious $3.3 \mu \mathrm{m}$ PAH emission present. The energetics are favorable for PAH emission, too: a main-sequence A2 V star supplies sufficient UV flux to energize PAHs enough to produce the $11.7 \mu \mathrm{m}$ light, even before adding any UV excess from active accretion. With these data we cannot conclusively resolve this issue. In order to further constrain the emission mechanism, we have obtained additional observations in a series of narrowband mid-IR filters with Michelle at Gemini, which will be presented in an upcoming paper (M. D. Perrin et al. 2006, in preparation).

\subsection{PDS 144 South}

Vieira et al.'s (2003) optical spectroscopy confirmed the presence of emission lines in the spectrum of PDS 144S, including $\left[\mathrm{S}\right.$ II] $\lambda \lambda 6717,6730$ and $\left[\mathrm{O}_{\mathrm{II}}\right] \lambda \lambda 3726,3728$, in addition to $\mathrm{H} \alpha$. Therefore, PDS $144 \mathrm{~S}$ is actively accreting and must necessarily possess circumstellar material of its own. Furthermore, we find that PDS 144S has almost 5 mag of IR excess at $K_{s}$ band, unambiguously indicating the presence of warm circumstellar dust. However, the SED alone cannot tell us the distribu- tion of that dust; models using either disks or spherical envelopes can both adequately reproduce dusty SEDs. Resolved imaging is necessary to break the degeneracies between model parameters (Chiang et al. 2001).

We do not resolve a disk around PDS 144S in any of these observations (although we remind the reader that our Keck data are saturated on PDS 144S in all but one wave band, preventing PSF subtraction and greatly degrading disk detection limits). Given PDS 144S's unresolved appearance, any disk around PDS 144S must be either noncoplanar with PDS 144N's disk or sufficiently less massive that it is optically thin at these wavelengths, so that the system's appearance is dominated by the direct light from the star. Jensen et al. (2004) observed 19 multiple T Tauri systems and found that eight out of nine binaries had polarization vectors aligned within $30^{\circ}$, consistent with disk alignment. However, these binaries have apparent separations of 200-1000 AU, much less than PDS 144's 5400 AU. It is plausible that disk alignment mechanisms may be less effective in systems with greater separations. HK Tau provides a well-known example of noncoplanar disks in a system apparently quite similar to PDS 144 (Stapelfeldt et al. 1998; Duchêne et al. 2003).

Unlike PDS 144N, PDS 144S displays no evidence for PAH emission above continuum levels in the present observations; the difference in the shapes of the two stars' SEDs from 3.6 to $8 \mu \mathrm{m}$ is quite striking. PDS 144S has sufficient far-UV flux to energize PAHs if present. Possibly, the two stars' disks differ in composition, with PDS 144S lacking PAHs. Alternatively, Acke \& van den Ancker (2004) suggested that Herbig Ae stars with flat disks have much weaker PAH emission than stars with flared disks because self-shadowing prevents UV light from reaching the PAHs. Thus, the two PDS 144 stars could have similar abundances of PAHs, yet very different spectra if their circumstellar dust geometries differ.

\section{PRELIMINARY DISK MODELS}

We have modeled the disk around PDS 144N using the Monte Carlo radiative transfer code made available by $\mathrm{B}$. Whitney and collaborators (Whitney et al. 2003a, 2003b). This was a firstdraft modeling effort only, with model parameters tuned by eye to approximate the observed properties of PDS 144 at $H$ band. No attempt has been made here to minimize errors in any formal statistical sense. The purpose of this exercise was to identify a reasonable starting point for future, more detailed modeling, and to determine which if any features of PDS 144N are most discrepant from simple disk models. A more sophisticated fit to the complete multiwavelength data set via a multidimensional optimization code will be described in a future work. Here we limit ourselves to discussing the overall properties of our model, and the most notable discrepancies between model and observation.

The Whitney et al. radiative transfer code allows grain properties to vary between different regions. Briefly, grains are treated as spheres with scattering computed using the Mie theory; grains follow power-law size distributions with parameters varying from ISM-like in the envelope and outflow to larger grains in the disk and midplane. We refer readers to Whitney et al. (2003a) for a detailed discussion of assumed grain parameters; we used a prescription identical to what those authors used. We assumed a disk mass of $0.01 M_{\odot}$, outer radius $r=350 \mathrm{AU}$, density exponent $\alpha=2.25$, scale height $h=0.4 \mathrm{AU}$ at $r=50 \mathrm{AU}$, and scale height exponent $\beta=1.25$.

We first attempted to model PDS $144 \mathrm{~N}$ using the simplest possible model of only a passive circumstellar disk, without any surrounding envelope. We did this computation for $H$ band, the wavelength in which the wings are least pronounced. The overall 


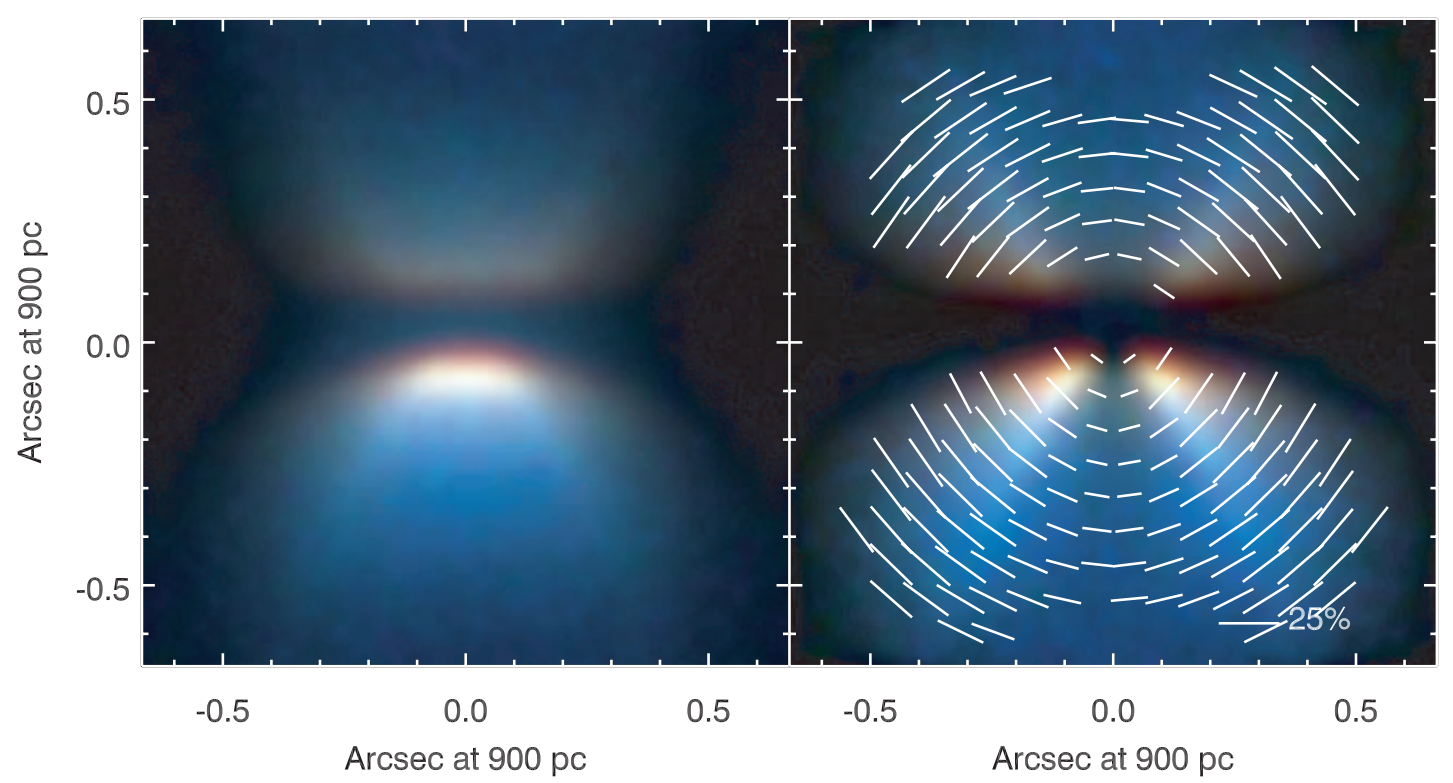

FIG. 6.-Monte Carlo model of a circumstellar disk plus envelope, at $1000 \mathrm{pc}$, convolved with a wavelength-dependent model Keck PSF computed with the Keck AO model of Sheehy et al. (2006). The left frame shows total intensity $I$, while the right frame shows polarized intensity $\left(Q^{2}+U^{2}\right)^{1 / 2}$. $H, K^{\prime}$, and $L^{\prime}$ are blue, green, and red, respectively, and the image scale is linear. Vectors in the right-hand frame show the degree and orientation of $K^{\prime}$-band polarization. The disk model has mass $0.01 M_{\odot}$ and radius $350 \mathrm{AU}$. Surrounding that is a rotationally supported infalling envelope (Terebey et al. 1984) with an infall rate of $2 \times 10^{-6} M_{\odot} \mathrm{yr}^{-1}$ and a cavity opening half-angle of $15^{\circ}$.

shape of the disk, including the slight curvature of the dark lane, is best reproduced by disk models with inclinations of $83^{\circ} \pm 1^{\circ}$. Despite an extensive search of parameter space, the simple disk models were unable to reproduce the extended nebulosity around the disk. Furthermore, although the wings are minimized at $H$ band, the northwestern lobe still has a local minimum on the vertical axis, while the disk-only models predict a local maximum there. Thus, we conclude that a circumstellar envelope is required in addition to a disk to accurately model this system.

Models incorporating a circumstellar envelope and outflow cavities are much more successful in reproducing the observed morphology. We added a rotationally supported infalling envelope (Terebey et al. 1984) with an infall rate of $2 \times 10^{-6} M_{\odot} \mathrm{yr}^{-1}$ and a cavity opening half-angle of $15^{\circ}$. We display this model in Figure 6. Limb brightening from the edges of the outflow cavity roughly reproduces the wings seen around PDS 144N.

However, there remain some discrepancies between this model and the data. While this is only a preliminary model, we point out some differences so that they may be addressed in future, more comprehensive modeling. The observed nebula has a sharper outer edge than the model does. This is not a $\mathrm{S} / \mathrm{N}$ effect: the observed nebula outer radius is roughly the same at all wavelengths, although the $\mathrm{S} / \mathrm{N}$ varies substantially. Furthermore, introducing read- and sky-subtraction noise comparable to the observed levels into our model images does not truncate the apparent size of the disk. We have discussed, above, possible scenarios for tidal or photoevaporative truncation of the material around PDS $144 \mathrm{~N}$. Also, the model predicts that for an inclination of $83^{\circ}$, the nearer side of the disk (southwest) will have a peak surface brightness $\sim 3$ times the fainter side. The observed southwest side is actually 10 times brighter than the fainter side. This difference may reflect the model's use of Mie scattering by spherical grains rather than more realistic grain scattering.

\section{CONCLUSIONS}

We have presented high-resolution observations of PDS 144 from 1.2 to $11.7 \mu \mathrm{m}$ obtained at Lick and Keck Observatories, plus archival Spitzer photometry from 3.6 to $70 \mu \mathrm{m}$. PDS $144 \mathrm{~N}$ is hidden from direct view at all wavelengths from 1.2 to $11.7 \mu \mathrm{m}$ by an edge-on, optically thick circumstellar disk 0.8 across. Red wings extend vertically from the disk, most likely due to scattering from the walls of an outflow cavity in an infalling envelope of dust and gas. The wings are very bright in the $3.3 \mu \mathrm{m}$ PAH feature, indicating the presence of small aromatic grains fluorescing due to far-UV illumination. Its nearby companion, PDS 144S, is unresolved in our data but has very strong IR excesses indicating that it, too, is surrounded by copious circumstellar dust. But a number of basic questions remain open about this fascinating system:

How far away is it? - Our distance estimate of $1000 \pm 200 \mathrm{pc}$ is based on fitting the UBVRI measurements of Vieira et al. (2003) with an extincted A5 stellar spectrum for PDS 144S. Uncertainties in the spectral type and evolutionary state of the stars, plus confusion of the two sources in the unresolved optical photometry, limit the precision possible with this method. The $1000 \mathrm{pc}$ distance also results in a surprising height above the Galactic plane. We caution that this distance estimate relies on the assumption that the two stars of PDS 144 are physically associated, which is probable but unproven.

How old is it? - The uncertain distance results in similarly uncertain total luminosities for the two stars, making it difficult to place them on pre-main-sequence evolutionary tracks (e.g., Palla \& Stahler 1993). The large IR excesses of both stars suggest that there may be significant accretion luminosity. This further complicates placing the stars on pre-main-sequence tracks, but also implies that the system is quite young.

How massive is it? - The stellar masses are poorly constrained for the same reason that their ages are, because their positions in the H-R diagram are imprecise. The dust mass around PDS 144N cannot be constrained with the data presented here, since the disk is optically thick at these wavelengths. Resolved millimeter observations of this system would be very useful to constrain the dust masses around each star. Given the southern declination, the Submillimeter Array (SMA) may be the array best suited to obtaining these observations. 
How have the two stars interacted? -PDS 144N's envelope appears to have been truncated, perhaps due to the influence of PDS 144S, although the mechanism for this truncation remains unclear. The two stars both have circumstellar material, but have very different appearances at these wavelengths. Can this be explained entirely by geometric effects due to different viewing angles to physically similar young stars, or are the stars and their circumstellar material intrinsically different? If so, why-given that they are presumably coeval, of fairly similar mass, and formed from the same parent cloud?

Are there outflows? - The presence of a bipolar cavity around PDS $144 \mathrm{~N}$ suggests that there are, or at least once were. Polar outflows from disks around $\mathrm{T}$ Tauri stars may be traced by a number of low-excitation emission lines, such as in the exquisite HST images of the HH 30 disk and jet (Burrows et al. 1996). Similar outflows have been seen around a number of Herbig Ae stars (Corcoran \& Ray 1998; Grady et al. 2000).

Does PDS $144 N$ also harbor such jets? - The presence of [S II] emission in the optical suggests that it does. At near-IR wavelengths at which AO imaging is possible, the [Fe II] 1.257 and $1.644 \mu \mathrm{m}$ lines are ideal outflow tracers on account of their low ionization potential and excitations (Pesenti et al. 2003; Pyo et al. 2003).

As the first Herbig Ae star with a clearly resolved edge-on disk, PDS $144 \mathrm{~N}$ provides an exciting opportunity for comparison of disk properties between intermediate-mass and lower mass stars, such as the well-studied HH 30. The geometry of the PDS 144 system makes it a particularly well suited laboratory for detailed studies of circumstellar material. Such studies need both high sensitivity and high angular resolution. Because the disk is edge on, it blocks the direct starlight, resulting in a greatly favorable contrast ratio and thus increased sensitivity to faint emission. Furthermore, since PDS 144 is a binary, we have a bright unocculted star conveniently nearby to serve as a wavefront reference source. Just as in HK Tau, the binarity of this system enables studies that would otherwise not be possible.

We greatly appreciate the excellent work of the Lick and Keck Observatory staffs, in particular Elinor Gates, Mark Kassis, and Randy Campbell. We also thank Bob Becker for his gracious flexibility in trading LWS observing time, and a \$2 bill. Sergio Vieira and Carlos Torres provided helpful discussions regarding their PDS survey observations. We recognize that Mauna Kea has always been a site of great significance to the Hawaiian people, and we are most fortunate to have the opportunity to observe from this mountain.

This work has been supported in part by the National Science Foundation Science and Technology Center for Adaptive Optics, managed by the University of California at Santa Cruz under cooperative agreement AST 98-76783. P. K. received additional support from the NASA Origins Program under grant NAG5-11769. M. D. P. is supported by a NASA Michelson Graduate Fellowship, under contract with the Jet Propulsion Laboratory (JPL). JPL is managed for NASA by the California Institute of Technology.

Facilities: Shane, Keck:I, Keck:II, Spitzer
Acke, B., \& van den Ancker, M. E. 2004, A\&A, 426, 151

Adams, F. C., Hollenbach, D., Laughlin, G., \& Gorti, U. 2004, ApJ, 611, 360

Ardila, D. R., Golimowski, D. A., Krist, J., Clampin, M., Ford, H. C., \& Illingworth, G. D. 2005, in Protostars and Planets V, ed. V. Mannings et al. (Houston: LPI), 8511

Artymowicz, P., \& Lubow, S. H. 1994, ApJ, 421, 651

Aumann, H. H., et al. 1984, ApJ, 278, L23

Bregman, J., Larson, K., Rank, D., \& Temi, P. 1994, ApJ, 423, 326

Burrows, C. J., et al. 1996, ApJ, 473, 437

Carballo, R., Wesselius, P. R., \& Whittet, D. C. B. 1992, A\&A, 262, 106

Cardelli, J. A., Clayton, G. C., \& Mathis, J. S. 1989, ApJ, 345, 245

Chiang, E. I., Joung, M. K., Creech-Eakman, M. J., Qi, C., Kessler, J. E., Blake, G. A., \& van Dishoeck, E. F. 2001, ApJ, 547, 1077

Cohen, M., Walker, R. G., Carter, B., Hammersley, P., Kidger, M., \& Noguchi, K. 1999, AJ, 117, 1864

Corcoran, M., \& Ray, T. P. 1998, A\&A, 336, 535

Cotera, A. S., et al. 2001, ApJ, 556, 958

D’Alessio, P., Calvet, N., \& Hartmann, L. 2001, ApJ, 553, 321

Dobashi, K., Uehara, H., Kandori, R., Sakurai, T., Kaiden, M., Umemoto, T., \& Sato, F. 2005, PASJ, 57, S1

Duchêne, G., Ménard, F., Stapelfeldt, K., \& Duvert, G. 2003, A\&A, 400, 559

Ducourant, C., Teixeira, R., Périé, J. P., Lecampion, J. F., Guibert, J., \& Sartori, M. J. 2005, A\&A, 438, 769

Elias, J. H., Frogel, J. A., Matthews, K., \& Neugebauer, G. 1982, AJ, 87, 1029

Evans, N. J., et al. 2003, PASP, 115, 965

Fazio, G. G., et al. 2004, ApJS, 154, 10

Galland, F., Lagrange, A.-M., Udry, S., Chelli, A., Pepe, F., Queloz, D., Beuzit, J.-L., \& Mayor, M. 2005, A\&A, 443, 337

García-Hernández, D. A., Manchado, A., García-Lario, P., Domínguez-Tagle,

C., Conway, G. M., \& Prada, F. 2002, A\&A, 387, 955

Gavel, D. T., et al. 2002, Proc. SPIE, 4494, 336

Grady, C. A., et al. 2000, ApJ, 544, 895

2001, AJ, 122, 3396

2005, ApJ, 630, 958

Habart, E., Natta, A., \& Krügel, E. 2004, A\&A, 427, 179

Habart, E., Natta, A., Testi, L., \& Carbillet, M. 2006, A\&A, 449, 1067

Herbig, G. H. 1960, ApJS, 4, 337

Herbst, W., \& Shevchenko, V. S. 1999, AJ, 118, 1043
Hernández, J., Calvet, N., Briceño, C., Hartmann, L., \& Berlind, P. 2004, AJ, 127,1682

Hillenbrand, L. A., Strom, S. E., Vrba, F. J., \& Keene, J. 1992, ApJ, 397, 613 Hollenbach, D., Johnstone, D., Lizano, S., \& Shu, F. 1994, ApJ, 428, 654

Jensen, E. L. N., Mathieu, R. D., Donar, A. X., \& Dullighan, A. 2004, ApJ, 600,789

Jones, B., \& Puetter, R. C. 1993, Proc. SPIE, 1946, 610

Kalas, P., Graham, J. R., \& Clampin, M. 2005, Nature, 435, 1067

Kroupa, P. 1998, MNRAS, 298, 231

Kuhn, J. R., Potter, D., \& Parise, B. 2001, ApJ, 553, L189

Leger, A., D'Hendecourt, L., \& Defourneau, D. 1989, A\&A, 216, 148

Lloyd, J. P., Liu, M. C., Macintosh, B. A., Severson, S. A., Deich, W. T., \& Graham, J. R. 2000, Proc. SPIE, 4008, 814

Makovoz, D., \& Marleau, F. R. 2005, PASP, 117, 1113

McCabe, C., Duchêne, G., \& Ghez, A. M. 2003, ApJ, 588, L113

Merín, B., et al. 2004, A\&A, 419, 301

Monin, J.-L., \& Bouvier, J. 2000, A\&A, 356, L75

Natta, A., \& Kruegel, E. 1995, A\&A, 302, 849

Padgett, D. L., Brandner, W., Stapelfeldt, K. R., Strom, S. E., Terebey, S., \& Koerner, D. 1999, AJ, 117, 1490

Palla, F., \& Stahler, S. W. 1993, ApJ, 418, 414

Perrin, M. D., Graham, J. R., Kalas, P., Lloyd, J. P., Max, C. E., Gavel, D. T., Pennington, D. M., \& Gates, E. L. 2004a, Science, 303, 1345

. 2004b, Proc. SPIE, 5490, 309

Persson, S. E., Murphy, D. C., Krzeminski, W., Roth, M., \& Rieke, M. J. 1998, AJ, 116, 2475

Pesenti, N., Dougados, C., Cabrit, S., O’Brien, D., Garcia, P., \& Ferreira, J. 2003, A\&A, 410, 155

Piétu, V., Dutrey, A., \& Kahane, C. 2003, A\&A, 398, 565

Pontoppidan, K. M., \& Dullemond, C. P. 2005, A\&A, 435, 595

Pyo, T., et al. 2003, ApJ, 590, 340

Rieke, G. H., et al. 2004, ApJS, 154, 25

Roe, H. G. 2002, Ph.D. thesis, Univ. California, Berkeley

Sheehy, C., McCrady, N., \& Graham, J. 2006, ApJ, in press (astro-ph/0604551)

Shu, F., Najita, J., Ostriker, E., Wilkin, F., Ruden, S., \& Lizano, S. 1994, ApJ, 429,781

Smith, B. A., \& Terrile, R. J. 1984, Science, 226, 1421

Stapelfeldt, K. R., Krist, J. E., Menard, F., Bouvier, J., Padgett, D. L., \& Burrows, C. J. 1998, ApJ, 502, L65 
Stapelfeldt, K. R., et al. 1999, ApJ, 516, L95

Staude, H. J., \& Neckel, T. 1992, ApJ, 400, 556

Stine, P. C., \& O’Neal, D. 1998, AJ, 116, 890

Teixeira, R., Ducourant, C., Sartori, M. J., Camargo, J. I. B., Périé, J. P., Lépine, J. R. D., \& Benevides-Soares, P. 2000, A\&A, 361, 1143

Terebey, S., Shu, F. H., \& Cassen, P. 1984, ApJ, 286, 529

Torres, C. A. O., Quast, G., de La Reza, R., Gregorio-Hetem, J., \& Lepine, J. R. D. 1995, AJ, 109, 2146

Vieira, S. L. A., Corradi, W. J. B., Alencar, S. H. P., Mendes, L. T. S., Torres, C. A. O., Quast, G. R., Guimarães, M. M., \& da Silva, L. 2003, AJ, 126, 2971
Waters, L. B. F. M., \& Waelkens, C. 1998, ARA\&A, 36, 233

Watson, A. M., \& Stapelfeldt, K. R. 2004, ApJ, 602, 860

Whitney, B. A., Wood, K., Bjorkman, J. E., \& Cohen, M. 2003a, ApJ, 598, 1079

Whitney, B. A., Wood, K., Bjorkman, J. E., \& Wolff, M. J. 2003b, ApJ, 591, 1049

Wilner, D. J., Holman, M. J., Kuchner, M. J., \& Ho, P. T. P. 2002, ApJ, 569, L115

Wizinowich, P., et al. 2000, PASP, 112, 315

Wolf, S., Padgett, D. L., \& Stapelfeldt, K. R. 2003, ApJ, 588, 373 